\title{
Load-dependent Path Planning Method for 3D Printing of Continuous Fiber Reinforced Plastics
}

\author{
Ting Wang ${ }^{\mathrm{a}}$, Nanya Li ${ }^{\mathrm{a} *}$, Guido Link ${ }^{\mathrm{a}}$, John Jelonnek ${ }^{\mathrm{a}}$, Jürgen Fleischer ${ }^{\mathrm{b}}$, Jörg Dittus ${ }^{\mathrm{b}}$, Daniel \\ Kupzik $^{\mathrm{b}}$.
}

\author{
a Institute for Pulsed Power and Microwave Technology, Karlsruhe Institute of Technology, \\ Eggenstein-Leopoldshafen, 76344, Germany \\ b wbk Institute of Production Science, Karlsruhe Institute of Technology, Karlsruhe,76131, \\ Germany \\ corresponding author: Nanya Li, nanya.li@kit.edu
}

\begin{abstract}
3D printing, to print continuous fiber reinforced plastics (CFRPs) has advantages of manufacturing complex shape and short production cycle. Due to anisotropic mechanical properties of continuous fibers, the paving direction of the fibers determines the mechanical strengths of the printed CFRPs. In this paper, a novel load-dependent path planning (LPP) method has been proposed to generate printing path for CFRPs, which exactly follows the load transmission path of the parts and could provide higher mechanical properties. A topology optimization method is applied to simplify the original disordered load distribution. In the developed Stress Vector Tracing (SVT) algorithm, the printing paths are generated along the load transmission path with the variable spacing of adjacent paths. The LPP method has been compared with the state-of-the-art printing path planning method for continuous fibers and shown better load-bearing and printability.
\end{abstract}

Key words: Load-dependent path planning; Continuous fiber reinforcement; Stress vector tracing; 3D printing.

\section{Introduction}

Fused filament fabrication (FFF) of thermoplastic materials has advantages of low costs and short production cycle [1]. Generally, filaments are extruded through a hot-end of a nozzle and deposited on a printing platform to build a geometry layer-by-layer [2]. Due to higher strength and lightweight features, the continuous fiber exhibits enormous potential to enhance the plastic parts [3, 4]. Three kinds of 3D 
printing technologies are commonly used in the manufacturing of continuous fiber reinforced polymer composites, as shown in Figure 1. For the methods exhibited in Figure 1(a) and (b), the composite filaments are impregnated while the printing process. The out-of-nozzle impregnation uses two feeders to simultaneously print the thermoplastic filament and continuous fiber on the printing bed [5]. The fusion of the continuous fiber and plastic are accomplished outside the nozzle. In the process of in-nozzle impregnation, the continuous fiber and thermoplastic filament are feed into a hot-end and mixed together inside [6]. In figure 1(c), a semi-finished CFRP filament has been fabricated as prepreg material and the heating cavity could be a conventional hot-end or a microwave resonant cavity [7]. The experimental results indicate that higher compressive properties can be obtained by using prepreg filament than other methods [8].

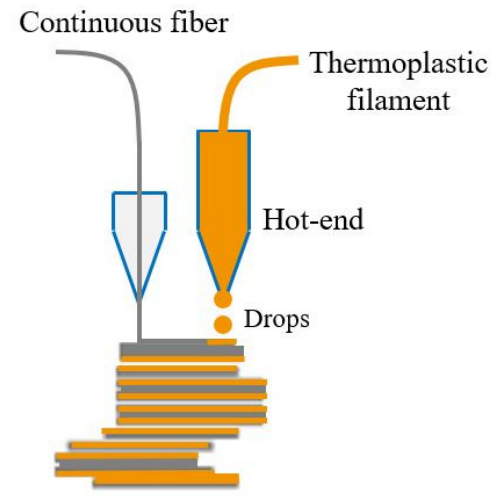

Out-of-nozzle impregnation

a

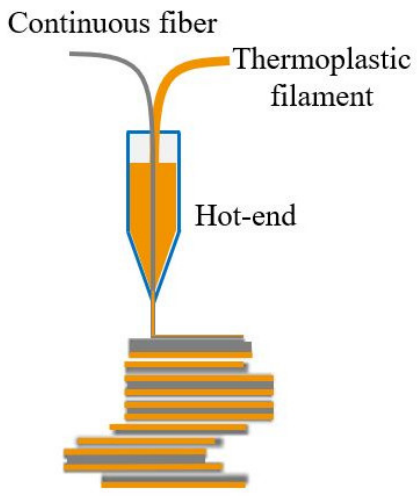

In-nozzle impregnation b

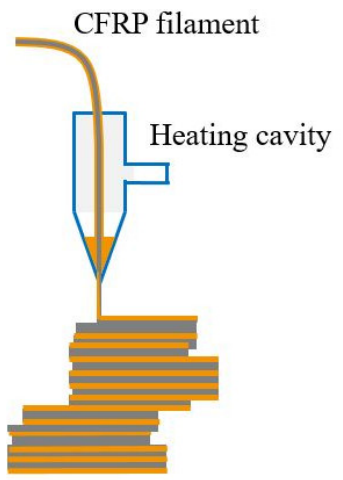

Semi-finished filament printing $\mathrm{c}$

Figure 1. Different 3D printing methods of continuous fiber reinforced plastics: (a) out-of-nozzle impregnation; (b) in-nozzle impregnation and (c) semi-finished CFRP filament printing.

Recently, the continuous fiber reinforced composites manufactured by FFF can only have fibers in simple geometry and limited areas, because of lacking effective path planning method [9]. As one of the key parameters, printing path has not only the influence on the geometry but also determines the mechanical properties of the printed part with different fiber orientations $[10,11]$. At present, the most commonly used printing patterns of CFRPs filaments FFF are raster path [12], zigzag path [13], Hilbertcurves path, spiral-curves path [14], contour-parallel path [15] and hybrid path [16], due to their simplicity and high adaptability. However, manufacturing complex components with CFPRs requires an uninterrupted and smooth printing path to avoid breakage of fibers and improve printing quality. 
Table 1. Comparison of different printing path planning methods of CFRPs.

\begin{tabular}{|c|c|c|}
\hline Printing path & Material & Author \\
\hline \multirow{2}{*}{ Straight line } & CF-PLA & Heidari-Rarani, et al. 2019 [12] \\
\hline & CF-ABS & Baumann, et al. 2017 [5] \\
\hline \multirow{3}{*}{ Zigzag } & CF-Nylon & Dutra, et al. 2019 [13] \\
\hline & CF-Nylon & Kousiatza, et al. 2019 [17] \\
\hline & CF-TPI & Ye, et al. 2019 [18] \\
\hline \multirow{3}{*}{ Contour } & CF-Nylon & Todoroki, et al. 2020 [19] \\
\hline & CF-Nylon & Mohammadizadeh, et al. 2019 [15] \\
\hline & CF-Nylon & Li, et al. 2016 [6] \\
\hline \multirow{2}{*}{ Zigzag-contour hybrid } & CF-Nylon & Naranjo-Lozada, et al. 2019 [16] \\
\hline & CF-Nylon & Ivey, et al. 2017 [20] \\
\hline \multirow{3}{*}{ Honeycomb } & CF-ABS & Ming, et al. 2019 [21] \\
\hline & CF-Nylon & Liu, et al. 2020 [22] \\
\hline & CF-PLA & Quan, et al. 2020 [23] \\
\hline \multirow{2}{*}{ Spiral } & CF -PLA & Akhoundi, et al. 2020 [14] \\
\hline & CF-Nylon & Dickson, et al. 2017 [24] \\
\hline Wavy & CF-Nylon & Adumitroaie, et al. 2019 [25] \\
\hline Grid & CF-Nylon & De Backer, et al. 2020 [26] \\
\hline 3D lattice & CF-Nylon & Eichenhofer, et al. 2017 [27] \\
\hline 3D lattice & CF-PLA & Liu, et al. 2018 [28] \\
\hline Curved path & CFRPs & Shembekar, et al. 2019 [29] \\
\hline \multirow{2}{*}{ Optimized based on stress } & CF-Nylon & Sugiyama, et al. 2020 [30] \\
\hline & CF-Nylon & Li, et al. 2020 [31] \\
\hline
\end{tabular}

CF: continuous fiber; ABS: acrylonitrile-butadiene-styrene; PLA: Polylactic acid; TPI: Thermoplastic-Polyimide.

The different 3D printing methods of CFRPs are compared and reviewed in Table 1. For example, Li et al. [6] researched the mechanical strength of the 3D printed CFRPs and used the zigzag-contour hybrid path to print the samples. Baumann et al. [5] compared the tensile properties of three different methods of producing fiber reinforced composites and used one-directional filling pattern to manufacture the testing samples. The contour-parallel printing pattern is also used in the manufacturing of the CFRPs testing samples [19]. Eiger software of Markforged uses zigzag-contour hybrid and concentric filling pattern for the printing process. Because the fiber reinforced filament provides excellent self-supporting property, the three-dimensional CFRPs can be printed. The printing paths optimized by stress distribution have been researched by Sugiyama et al. [30] and Li et al. [31], the preliminary research works show promising advantages to print the continuous carbon fibers along the load transmission path.

The above mentioned research achievements prove the considerable efforts in the ongoing development of 3D printing CFRPs. However, the path planning methods need further development to: 1) have an algorithm to match the printing path with load transmission; 2) generate continuous printing path to avoid damaging the continuity of stresses; 3) prevent small radius (high curvature) corner and sharp 
angle of the printing path which may break the continuous fiber. In this paper, a load-dependent path planning (LPP) method is developed under the consideration of principal stress distribution, fiber limitation and variable printing speed demands. Based on the topology optimization and developed Stress Vector Tracing (SVT) algorithm, the geometry is fully printed with a continuously connected fiber path. Comparisons have been done between the LPP, commercial path planning software (Slic3r) and the stateof-art path planning method of CFRPs.

\section{Load-dependent Path Planning (LPP) Method}

\subsection{Simplifying load distribution by topological optimization}

The ply direction of continuous fibers has a significant influence on the mechanical properties of manufactured CFRPs. Apparently, the printing path of continuous fiber reinforced thermoplastic filaments play an important role in controlling the mechanical properties of printed composites $[32,33]$. However, the 3D printing path planning of continuous fiber reinforced plastics has encountered bottleneck of parts with complex geometries, because of the disordered stress distribution under different load conditions. The disorganized and interrupted tensile and compressive stresses cannot be followed with a continuous path. Here, we involve a topological optimization method named Solid Orthotropic Material with Penalization (SOMP) [34] to re-arrange the original stress vectors in order. The SOMP topology optimization approach is based on the Solid Isotropic Material with Penalization (SIMP) approach, which is extended to the orthotropic material. The mechanical properties of a composite laminate [35] has been used during the topological optimization (Young's modulus: $E_{1}=18.3 \mathrm{Gpa}$;

$E_{2}=19.8 \mathrm{Gpa}$, Poisson's ratio: $v_{12}=v_{21}=0.17$, Shear modulus: $\left.G_{12}=5.15 \mathrm{Gpa}\right)$. The topology optimization method with design goal of minimum compliance can be written as [36]:

$$
\begin{aligned}
& \min : c(\rho, \theta)=U^{T} K U=\sum_{e=1}^{N}\left[T_{\min }+\left(\rho_{e}\right)^{P}\left(T_{0}-T_{\min }\right)\right] u_{e}^{T} k_{\theta}^{e} u_{e} \\
& \text { subjected to }:\left\{\begin{array}{l}
K U=F \\
\frac{V(\rho)}{V^{*}} \leq f \\
\rho \in[0,1]
\end{array}\right.
\end{aligned}
$$

Where $\rho$ orientation, $N$ $\rho_{e}$

U 
material. $T_{\min }$ is set to $10^{-3}$ in order to prevent any possible singularity of the equilibrium problem. $u_{e}$ and $k_{\theta}^{e}$ represents the element displacement vector and the element stiffness matrix. $V(\rho), V^{*}$ and $f$ are the material volume, design material volume and defined volume fraction respectively.

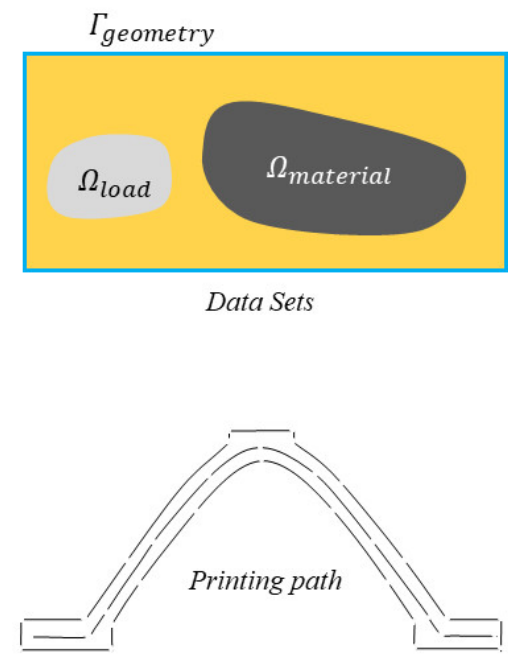

Path Reconfiguration and Connection

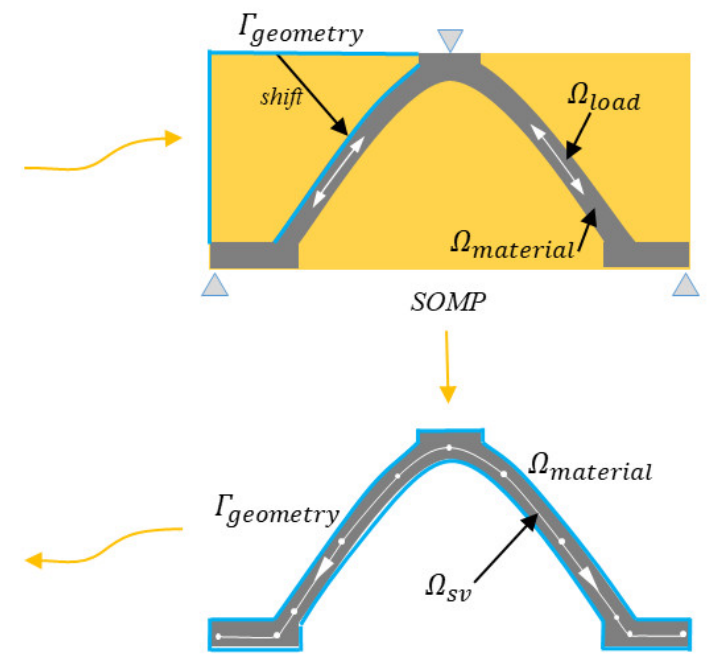

Stress Vector Tracing (SVT)

Figure 2. Schematic of the load-dependent path planning method.

As shown in Figure 2, the original design contains several data sets and boundary, including the material set ( $\left.\Omega_{\text {material }}\right)$, load set $\left(\Omega_{\text {load }}\right)$ and geometry boundary $\left(\Gamma_{\text {geometry }}\right)$. After the topological optimization process, the geometry boundary will shift to the new areas, which have higher stress density. The material set will reshape to a structure that only has beams and scaffolds under certain external loads. Meanwhile, the tensile and compressive stress vectors can be obtained. Then, a developed stress vector tracing algorithm (detailed information is described in section 2.2) has been used to define new geometry boundaries and coordinate the stress vector set $\left(\Omega_{s v}\right)$. A load-dependent printing path of continuous fiber reinforced plastics can be generated to have the fiber orientation follow the distribution of principal stresses.

\subsection{Developing Stress Vector Tracing (SVT) algorithm}

The SVT algorithm contains medial axis extraction by using Voronoi diagram [37] and vector-trace algorithm to obtain the printing paths which follow the load transmission. The medial axis extraction is to acquire geometrical features of the structure, as shown in Figure 3. The boundary points of the structure are used as the seeds of Voronoi diagram so that the adjacent edges of sub-regions can be extracted as 
medial axis, and then build a relationship between the medial axis points and geometrical boundary points. The yellow points are the geometrical boundary points, and for each two neighbor boundary points, an equidistant line called Voronoi edge is generated to divide the area between them, as shown in Figure 3 (a). With the segmentation of the Voronoi edges, the medial axis is generated and represented by the black curve located between the geometrical boundary curves, as shown in Figure 3 (b).

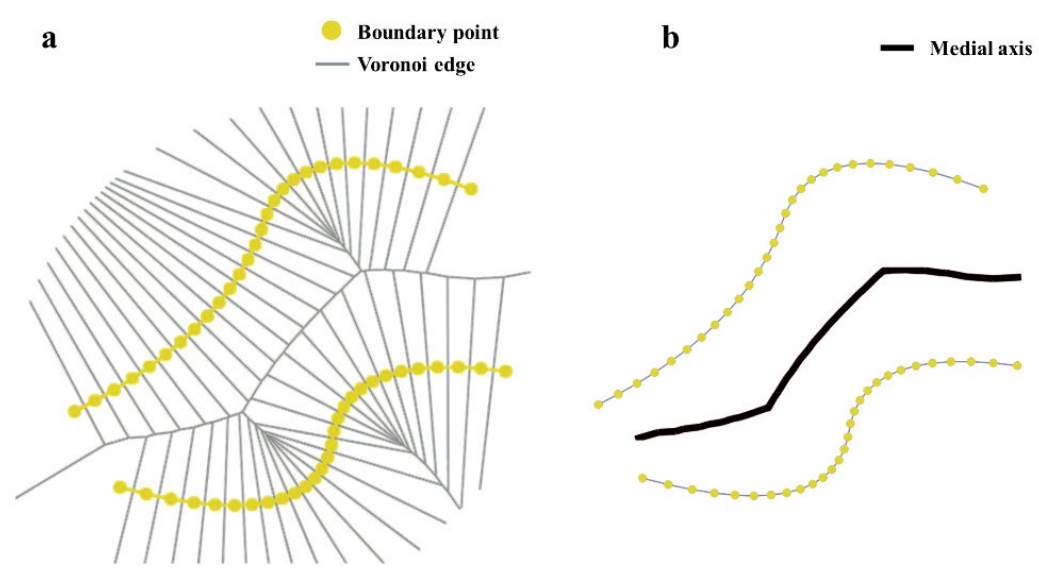

Figure 3. Schematic of using Voronoi diagram to generate medial axis: (a) Voronoi diagram; (b) generated medial axis.

There are three geometrical parameters that are used in the vector-trace algorithm, as shown in Figure 4. The angle $\beta$ between path segment and nearby stress vector. The $\alpha$ is the angle between two adjacent path segments, and $d$ is the distance between two nearby points. The physical meanings of these parameters represent the matching rate of the generated path and the stress vectors, the smoothness of the path and the resolution of the generated path based on the points of medial axis. Obviously, more points of the medial axis are used, the higher resolution of the generated path is.

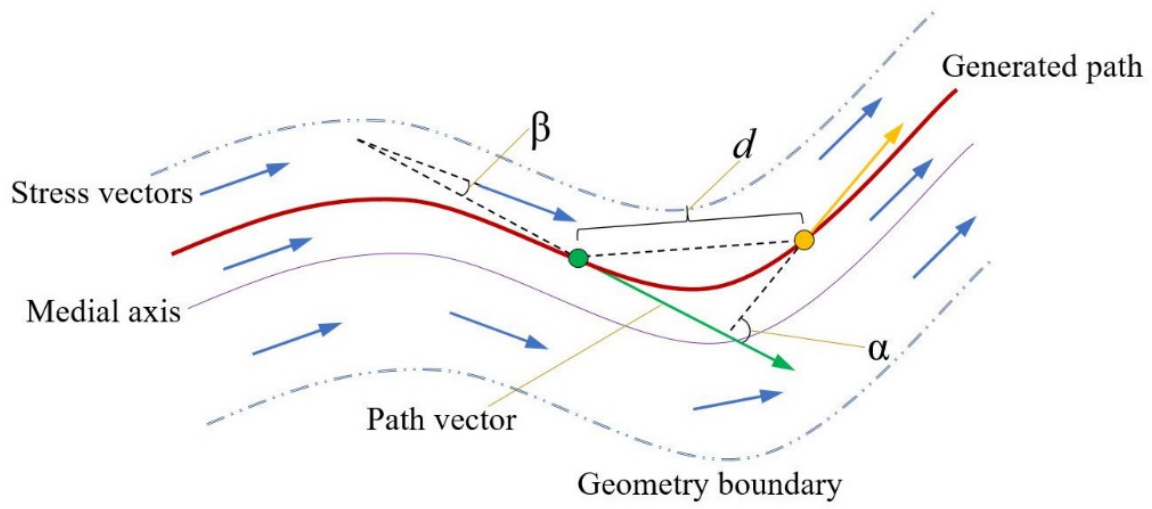

Figure 4. Geometrical parameters that are used in vector-trace algorithm. 
Ideally, the three geometrical parameters should as small as possible. Thus, an evaluation criterion has been applied to optimize these parameters. Assuming that the green path vector in Figure 4 is $v_{p l}$, and the angle $\alpha$ between the $v_{p n}$ (the next yellow vector) and $v_{p l}$ is calculated in Equation (2). The angle $\beta$ between stress vector ( $v_{\text {stress }}$ ) and $v_{p n}$ is calculated by Equation (3). And the distance $d$ between two nearby points on the path is also calculated.

$$
\begin{aligned}
& \alpha=\cos ^{-1}\left(\frac{v_{p l} \cdot v_{p n}}{\left|v_{p l}\right|\left|v_{p n}\right|}\right) \\
& \beta=\cos ^{-1}\left(\frac{v_{p n} \cdot v_{\text {stress }}}{\left|v_{p n}\right|\left|v_{\text {stress }}\right|}\right)
\end{aligned}
$$

There are several factors in the evaluation criterion that should be applied, including distance coefficient $\gamma(d)$, stress coefficient $\gamma(\beta)$ and path coefficient $\gamma(\alpha)$.They are uniformly expressed by $\gamma(z)_{z=\alpha, \beta, z}$ in the Equation(4), where the $k_{z}$ is the amplification factor (weight factor) according to different coefficient, the $\eta$ and $\varepsilon$ are arbitrary constants that are used to change each coefficient. By multiplying the three coefficients, the coefficient of smooth $\delta$ of each point can be obtained, as shown in Equation (5). The coefficient of smooth of generated path has been evaluated by considering turning angle, the difference between the path and stresses, and distance between two path points. Then the point with the smallest coefficient of smooth is chosen to be the next point of the path. The algorithm is executed until there are no more medial axis points left or the search area is empty.

$$
\begin{aligned}
& \gamma(z)_{z=\alpha, \beta, d}=\left(\frac{z}{z_{\text {limit }}}\right) \cdot \frac{\left(k_{z} \cdot \eta-\varepsilon\right)}{k_{z}}+\varepsilon \\
& \delta=\prod_{\mathrm{z}=\alpha, \beta, \mathrm{d}} \gamma(z)
\end{aligned}
$$

By using the SVT algorithm, the LPP with variable spacing are generated along the load transmission path. Based on the geometrical characteristics of the printing path, different printing speeds are applied. For example, the straight lines have the highest printing speed, the curved turns should be printed with low speed, to make sure the filament can continuously adhere to the printing bed. 


\section{Result and discussions}

\subsection{LPP of CF reinforced bridge and suspension parts}

In this section, a continuous fiber reinforced three-points-bending bridge and a suspension part are calculated to verify the developed path planning method. For the bridge part (width: $185 \mathrm{~mm}$ and height: $50 \mathrm{~mm}$ ), an external force and two support conditions are applied as shown in Figure 5 (a). After the mechanical analyzing, the stresses distribution is reorganized (Figure 5 (b)) and the tensile and compressive stresses can be separated into different areas and always along the medial axis direction of these beams, as illustrated in Figure 5 (c).

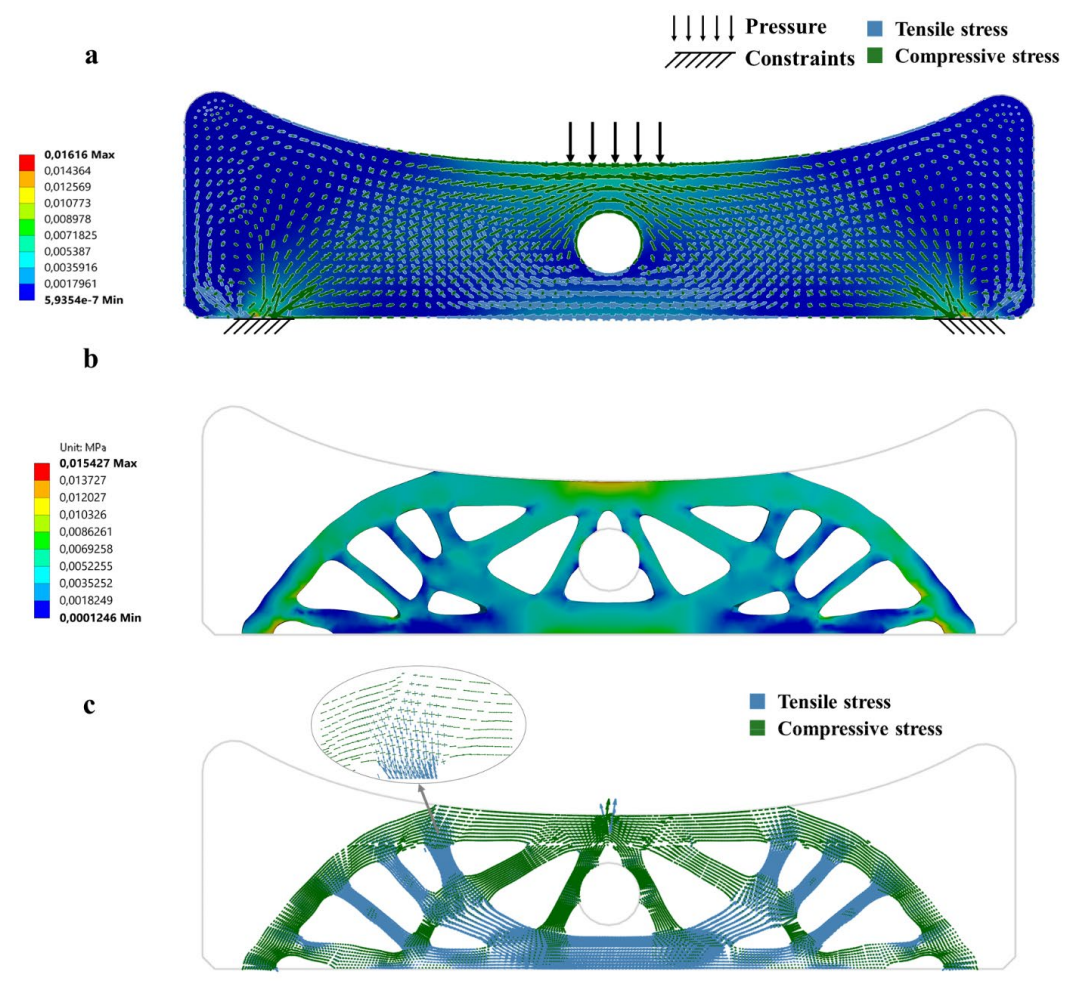

Figure 5. Stresses analyzing of the bridge part: (a) mechanical analysis model; (b) stresses distribution after analyzing; (c) re-ordered stresses distribution inside the part.

With the help of the developed vector-trace algorithm, the tensile and compressive stresses are calculated as load paths based on the medial axis. The printing paths with variable spacing are generated inside the geometrical boundaries and connected. The geometrical adaptive printing speeds according to the curvature, angle and length of straight lines (or curves) of the printing path are shown in Figure 6 . The parameters that are used to determine the printing speed of the printing path segments are shown in Table 2. 
Table 2. Printing speeds according to the curvature, length of the curves and angle.

\begin{tabular}{|l|l|l|l|}
\hline Printing speed & $7 \mathrm{~mm} / \mathrm{s}$ & $15 \mathrm{~mm} / \mathrm{s}$ & $35 \mathrm{~mm} / \mathrm{s}$ \\
\hline Curvature $\rho\left(\mathrm{mm}^{-1}\right)$ & $\rho>0.2$ & $0.05<=\rho<=0.2$ & $\rho<0.05$ \\
\hline Length L $(\mathrm{mm})$ & $\mathrm{L}<15$ & $15<=\mathrm{L}<=25$ & $\mathrm{~L}>25$ \\
\hline Angle A $\left(^{\circ}\right)$ & $\mathrm{A}<90$ & $90<=\mathrm{A}<=135$ & $\mathrm{~A}>=135$ \\
\hline
\end{tabular}

With regarding to the curvatures of the printing path, the curvature of each point on the path has been calculated based on the angle and distance between two adjacent directional vectors. The length of the curves is also an influencing factor, because some of the curves are not long enough to accomplish the acceleration and deceleration process. Thus, a high printing speed can only be arranged to a long distance and low curvature curve without sharp turn.

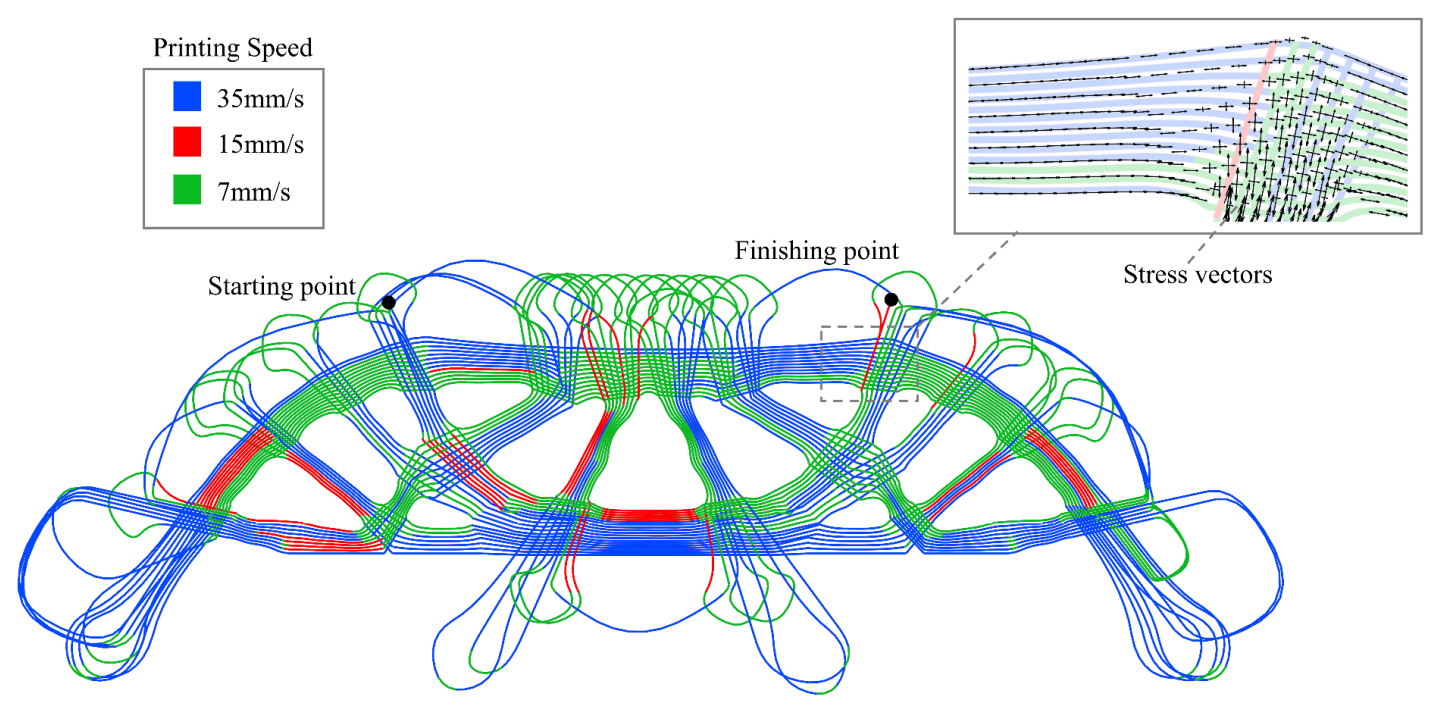

Figure 6. Continuously connected printing path of the bridge part with variable printing speeds.

In addition, it can be seen in the Figure 6 that the load-dependent path has less curves with high curvature and they are generally located outside of the geometry boundaries, which have no effect to the printed part. The curves inside the boundary have lower curvature because of the continuity of loadtransmission path. That indicates the load-dependent printing path is smoother than other commercial printing paths. As shown in Figure 7, a suspension part is studied to generate the load-dependent printing path and will be compared with the commercial path planning method. The suspension part has a $175 \mathrm{~mm}$ width, $126 \mathrm{~mm}$ height, and both of the paths of the bridge and suspension part can be shrunk or magnified. A compressive load is applied on the top of cantilever and bottom areas are fixed with 
supports. The stress analysis before and after the topology optimization are shown in Figure 7 (a) and (b). The main paths along the stresses transfer are generated by using the vector-trace algorithm and some segments of path are separated into different branches due to the different load-transmission directions, the result is shown in Figure 7 (c). Thus, the non-isometric printing paths in terms of the extracted loaddependent paths are generated and connected together, as shown in Figure 7 (d).
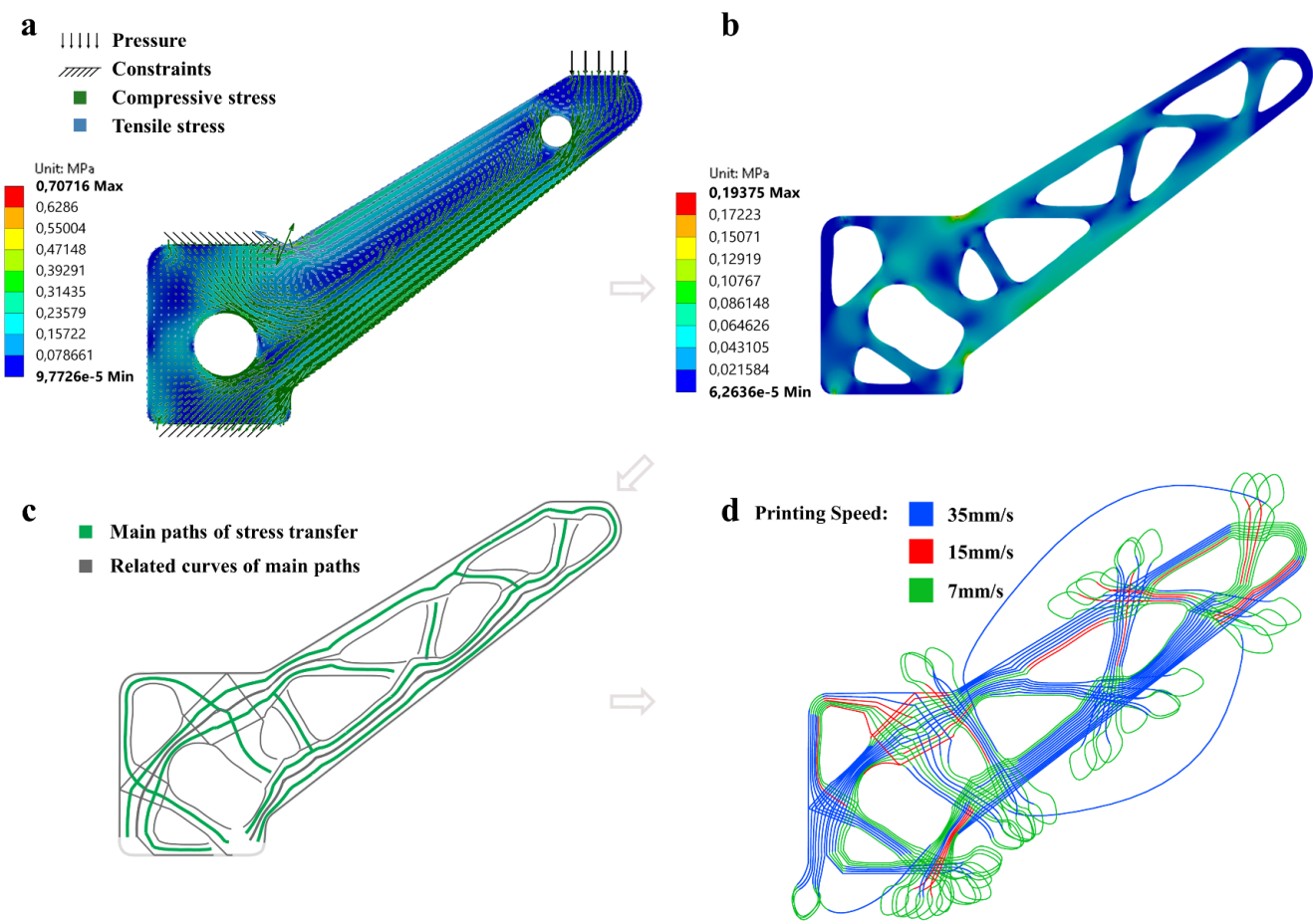

Figure 7. Processes of generating the continuous printing path of the suspension part: (a) load and stresses distribution; (b) optimized stresses distribution and area; (c) main printing paths generated by the developed vector-trace algorithm; (d) generated continuous printing paths.

\subsection{Comparison of load-dependent printing path with Slic3r}

The curvature of the printing path is a significant parameter for the $3 \mathrm{D}$ printing of CFRPs which affects directly the processing feasibility and quality of the printed part. The curvature of different printing paths of bridge part and suspension part are estimated and compared, as shown in Figure 8 and 9 respectively. The figure 8 (a) shows the curvature distribution of the LPP printing path, and Figure 8 (b) to (d) exhibit the curvature distributions of the concentric, $45^{\circ}$ zigzag and $90^{\circ}$ zigzag printing paths generated by Slic3r. All of the printing paths have the same highest value of the curvature and are about 4 $\mathrm{mm}^{-1}$. The concentric printing path shown in Figure 8 (b) has many sharp curves at turning points and the curvature distribution of the $45^{\circ}$ zigzag printing path and $90^{\circ}$ zigzag printing path own the same tendency 
(shown in Figure 8 (c) and Figure 8 (d)), due to the huge amount of turns with high curvature and abrupt printing direction variation. The LPP printing path is generated by following the principal stress transfers, which is smooth due to the integrity of the force transmission path so that it has a few high curvature curves. About $80 \%$ of the curves of the LPP printing path have curvature less than $0.26 \mathrm{~mm}^{-1}(3.8 \mathrm{~mm}$ radius of curvature).

a
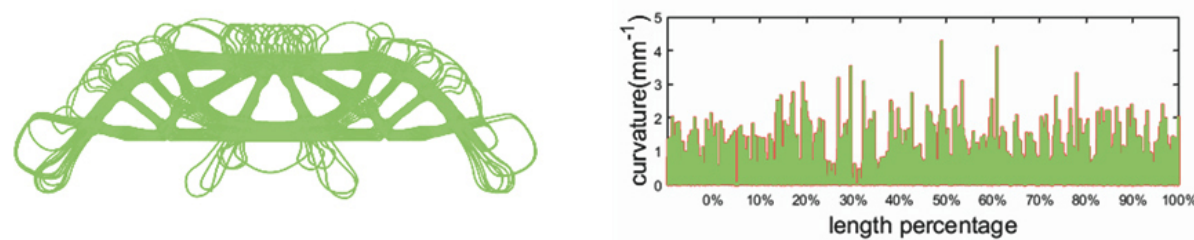

b
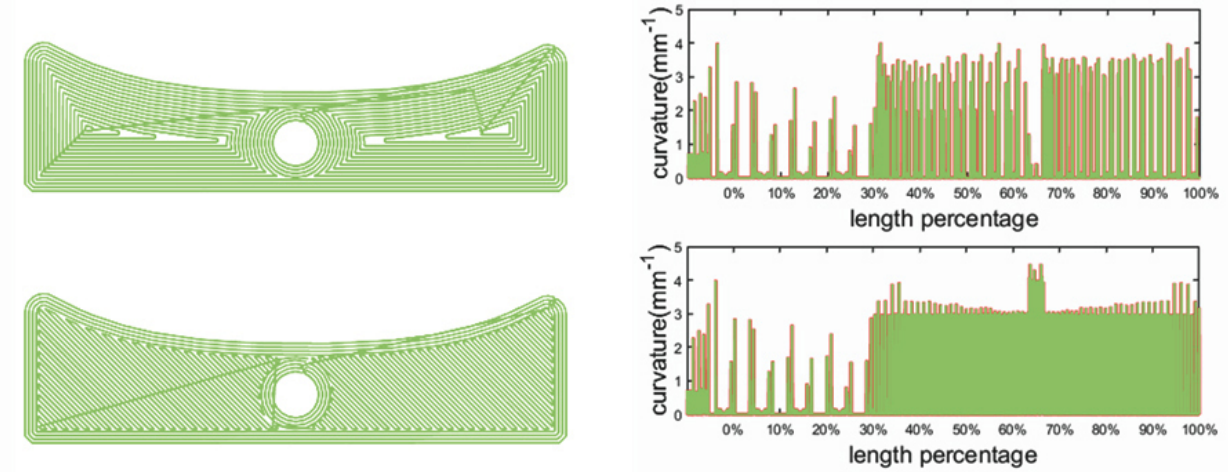

d
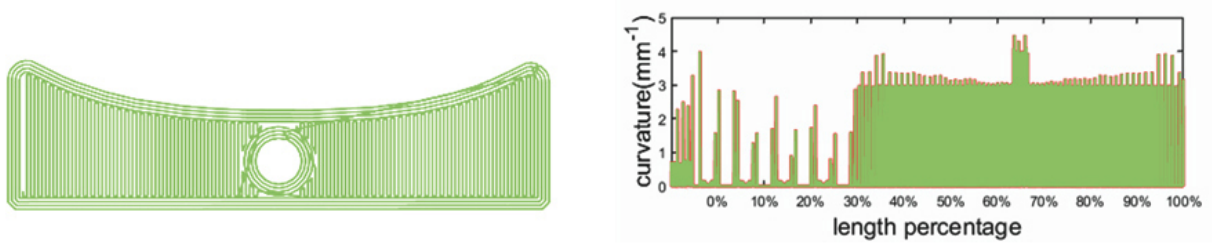

Figure 8. Curvature distribution of different printing paths of the bridge part: (a) to (d) curvature distribution of LPP, concentric, $45^{\circ}$ zigzag and $90^{\circ}$ zigzag printing paths.

Furthermore, the curvature distributions of different printing paths of suspension part are shown in Figure 9 (a) to (d), a similar result as that of the bridge part can be obtained. Significantly, the LPP printing path has only a few of high curvature curves that reach the highest value, in which about $72 \%$ of the curves have curvature less than $0.26 \mathrm{~mm}^{-1}$. Meanwhile, many curves of the printing paths generated by Slic3r have reached the highest value $4 \mathrm{~mm}^{-1}$. The high curvature curves of the concentric printing path are mostly located on the areas around the two holes. Moreover, the fluctuation of the curvature change of the LPP printing path has lower value and frequency than the other printing paths. 
a
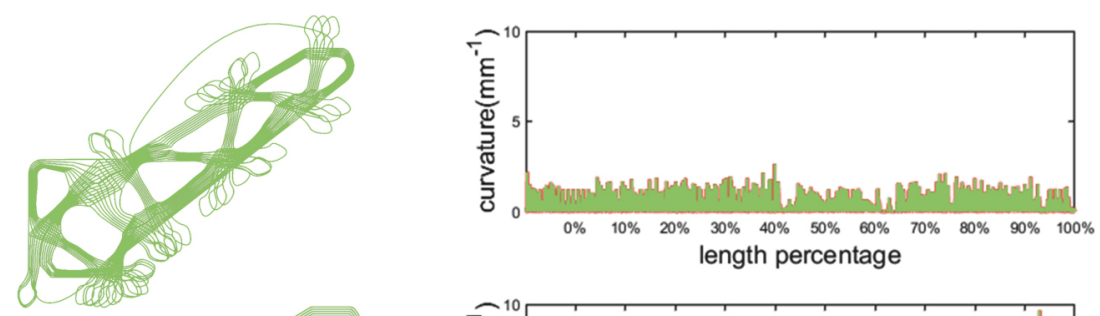

b
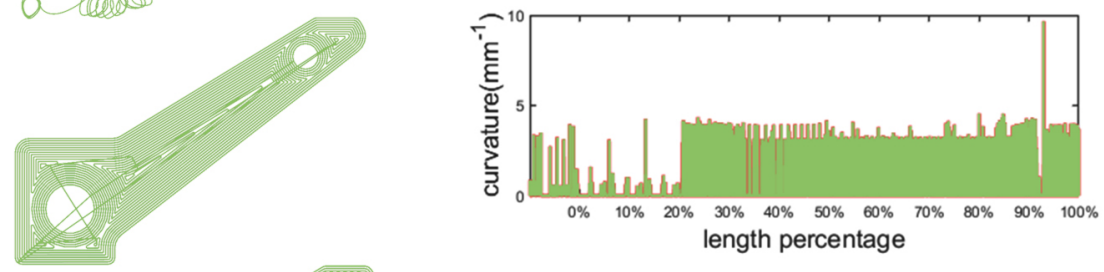

c
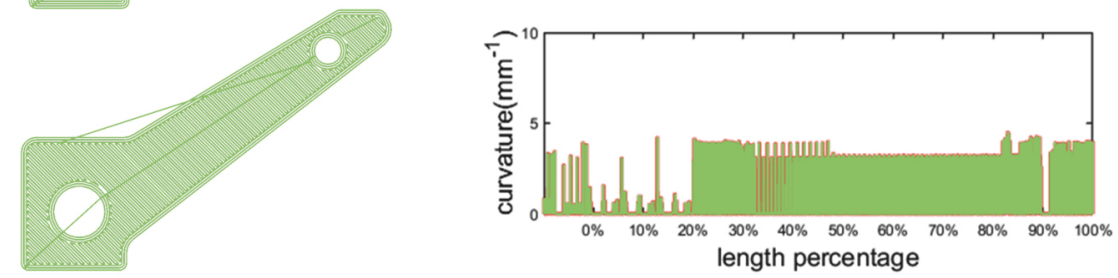

d

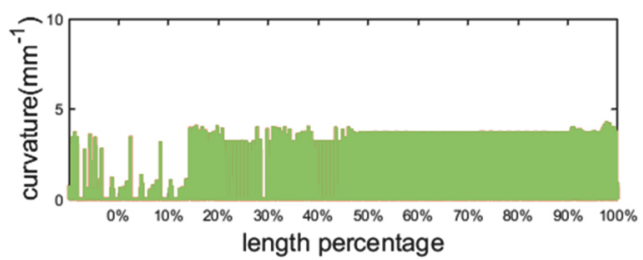

Figure 9. Curvature distribution of different printing paths of the suspension part: (a) to (d) curvature distribution of the LPP, concentric, $45^{\circ}$ zigzag and $90^{\circ}$ zigzag printing paths.

Another vital parameter of the generated printing path of CFRPs is the fiber orientation. The orientation difference between the principal stresses and printing path is represented by the angle between them. By counting how many path vectors are in the range of angle difference with adjacent stress vectors, the matching rate between the printing path and the stress distribution can be generally estimated. As shown in Figure 10, the LPP printing path of the bridge part has the highest matching rate than the other printing paths generated by Slic3r. As a result of the diverse filling patterns of the printing paths generated by Slic3r, the matching rate of each printing path is different. With reference to the stress distribution of the original bridge part (shown in Figure 5 (a)), the direction of the principal stress vectors around the hole is distributed along the boundary. Thus, the $45^{\circ}$ zigzag printing path accidentally has some paths that fit the load transmission paths. On the contrary, the $90^{\circ}$ zigzag and concentric printing paths can hardly match the stress vectors. 


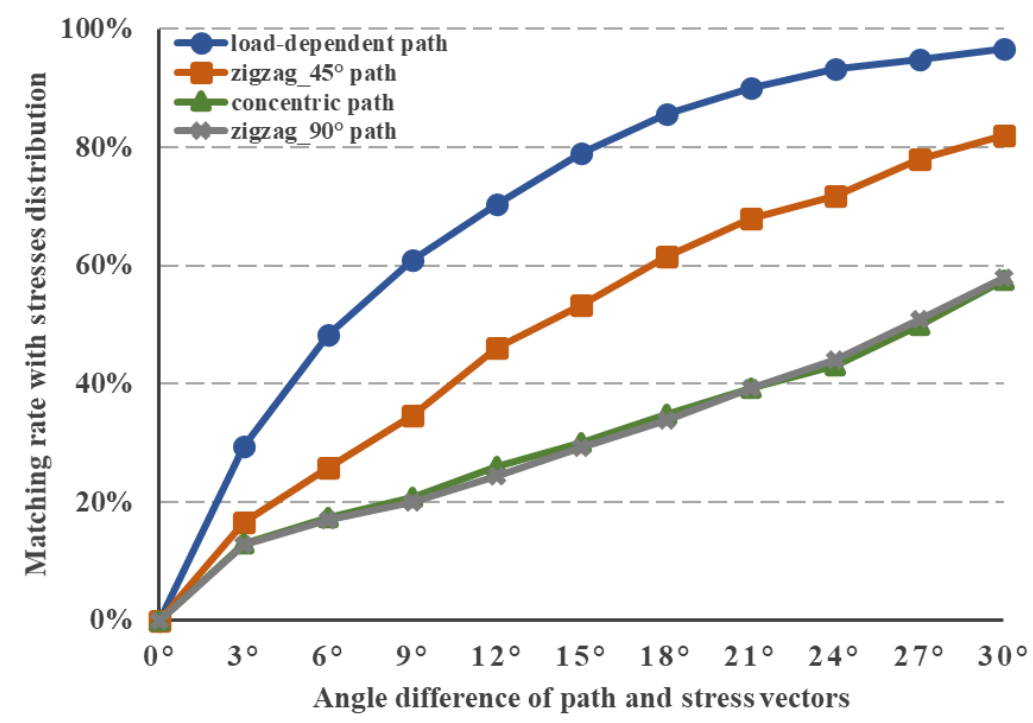

Figure 10. Matching rate of different printing paths of the three-points-bending bridge part according to various angle difference of path and stress vectors.

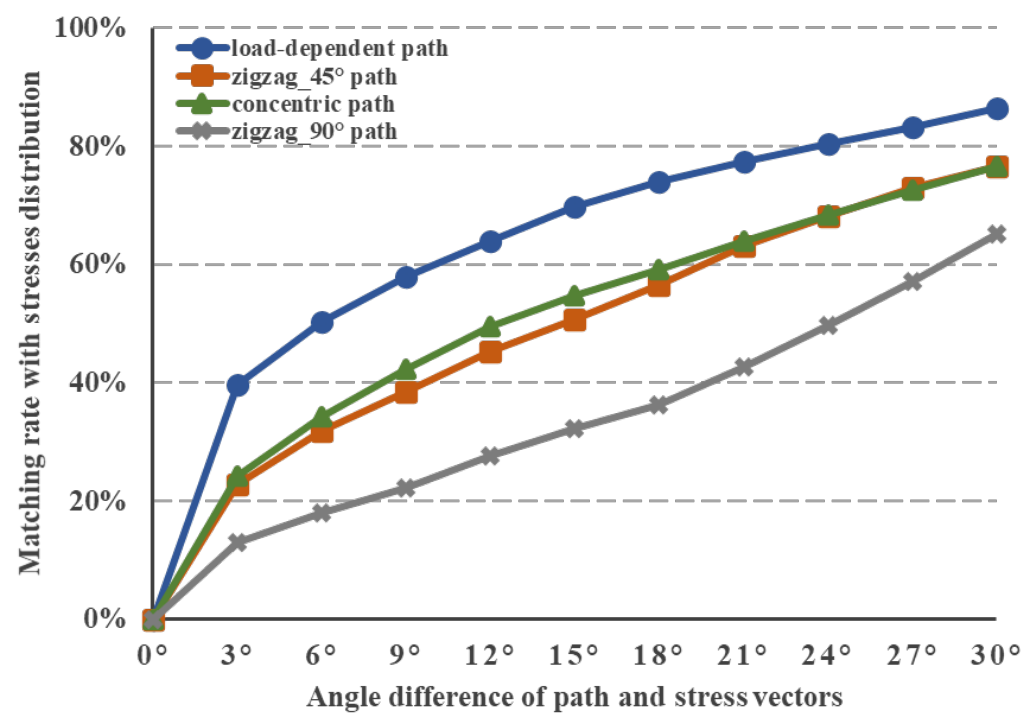

Figure 11. Matching rate of different printing paths of the suspension part according to various angle difference of path and stress vectors.

With the increase of angle difference, the matching rate of the LPP path is increased progressively. The variable spacing between adjacent paths allows the generated paths to follow the principal stresses adaptively. The other printing paths generated by Slic3r are partially matched with the stress distribution because some sporadic principal stresses vectors have a small angle difference with the nearby printing path by coincidence. It is worth to be mentioned that for the path of the three-points-bending bridge part 
about $96.6 \%$ of the principal stress vectors of the LPP path have less than $30^{\circ}$ angle difference with the printing path. As shown in Figure 11, the LPP path of the suspension part has about 20\% higher matching rate with the stress vectors than other printing paths of Slic3r. Meanwhile, the $90^{\circ}$ zigzag printing path has nearly no congruity with the principal stress distribution and shows the lowest matching rate.

\subsection{Comparison of LPP with state-of-the-art path planning method of CFRPs}

Currently, several path planning methods for topology optimized structures are developed and discussed by researchers. The printing paths of a topology optimized cantilever beam generated by using streamline method, offset method and Equally-Space method (EQS) are shown in Figure 12 (b), (c) and (d). For this cantilever beam, the left side is fixed and a load that points to the ground is applied on the right side of the beam, as shown in Figure 12 (a). Obviously, the offset method generated interrupted paths, which cannot be used for continuous fiber printing. Meanwhile, the path contains too many sharp turns, which may lead to a high possibility of fiber fracture. The generated printing paths of streamline and EQS methods (Figure 12 (b) and (d)) have smooth turns and continuous load transfer benefits, but the joint areas have no connections. The joint area, such as the middle of the cantilever beam, exactly indicates a complex stress distribution and requires printing paths from different directions.

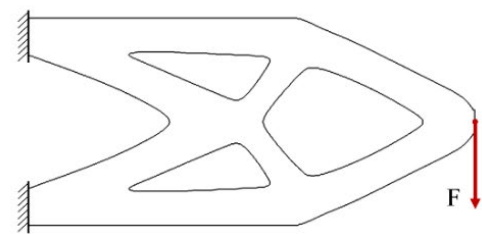

$\mathbf{a}$

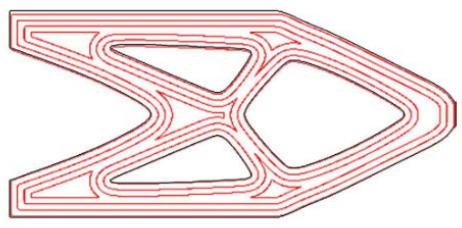

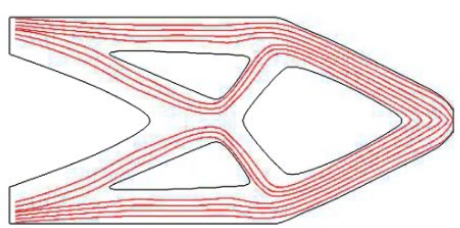

$\mathbf{b}$

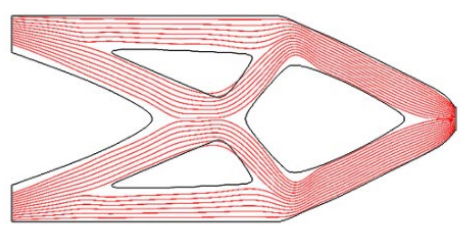

d

Figure 12. Printing paths of topology optimized cantilever beam [36]: (a) load and supports condition; (b) path generated by streamline method; (c) path generated by offset method; (d) path generated by EQS method.

As shown in Figure 13 (a), under the load conditions mentioned above, two different kinds of stresses can be found inside the part. The blue one is the tensile stresses and the green one is the compressive stresses. The medial axis of the structure has been extracted by using the stress vector tracing algorithm 
and marked in the figure. The load-dependent printing path generated by LPP has shown in Figure 13 (b), and it is obvious that the geometrical edges have been followed with same shape and the printing path can pave the continuous fiber along the two stress vectors without compromise. The important joint areas are covered by the paths that go cross each other to achieve a better bonding and reinforcing properties. That means the joint areas have higher degree of compaction than other areas, due to the same layer thickness requirement of printing process.

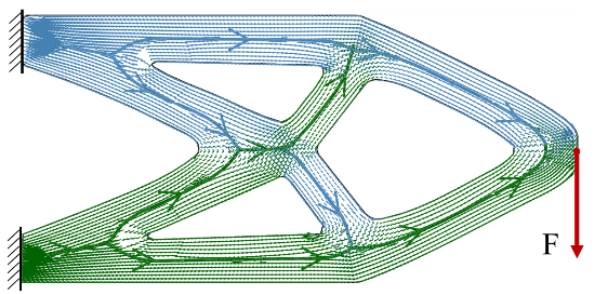

$\mathbf{a}$

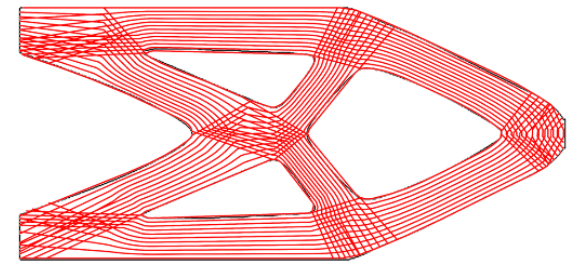

b

Figure 13. LPP generated printing paths of topology optimized cantilever beam: (a) main transfer directions of the stress distribution; (b) LPP generated printing path based on stress distribution.

Without loss of generality, an M-shape and an L-shape part are also evaluated and discussed. For the M-shape part, the left and right sides of the bottom are fixed and a force is applied in the middle, as illustrated in Figure 14 (a). Meanwhile, the top side of the L-shape part is fixed and a force is applied on the middle point of the right side, as shown in Figure 16 (a). The printing paths of these two parts generated by EQS, streamline and Offset methods are shown in Figure 14 and Figure 16, respectively.

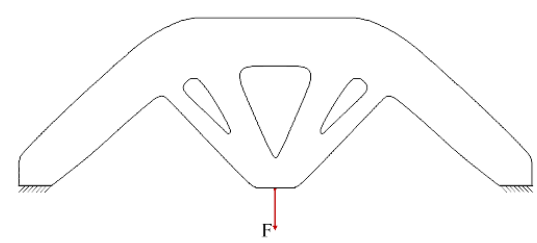

a

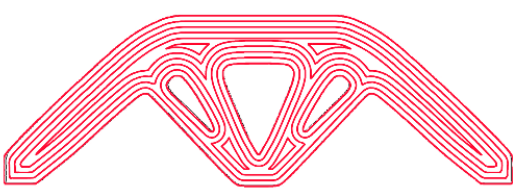

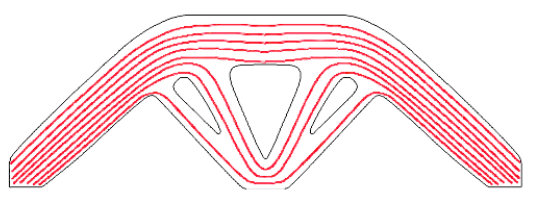

b

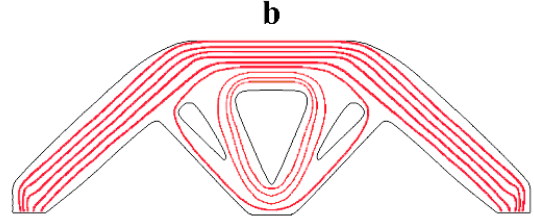

d

Figure 14. Printing paths of topology optimized M-shape part [36]: (a) applied point load and supports condition; (b) path generated by EQS method; (c) path generated by offset method; (d) path generated by streamline method. 
The printing paths of the M-shape part generated by the EQS method and streamline method can hardly fill the shape, especially at the corners, and the discontinuous offset printing path has too many sharp turns. As shown in Figure 15 (b), the LPP generated printing path has fully occupied the shape and are distributed along the principal stress transfer direction. The blue and green arrows in Figure 15 (a) represent the main distribution directions of the tensile and compressive stresses.

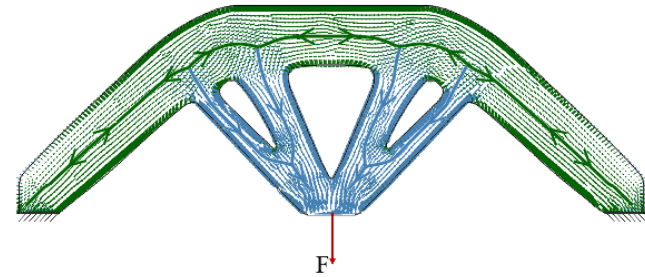

a

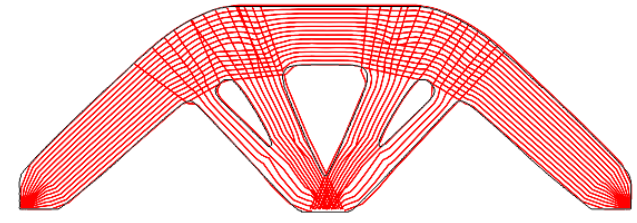

b

Figure 15. LPP generated printing paths of topology optimized M-shape part: (a) main transfer directions of the stress distribution; (b) LPP generated printing path based on stresses distribution.

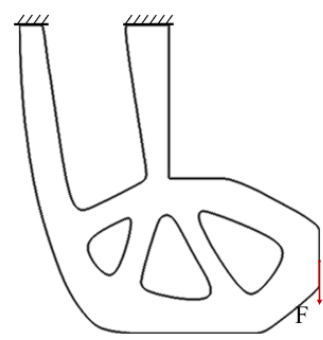

$\mathbf{a}$

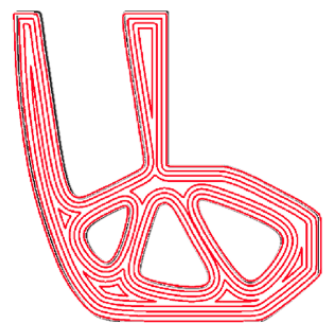

$\mathbf{C}$

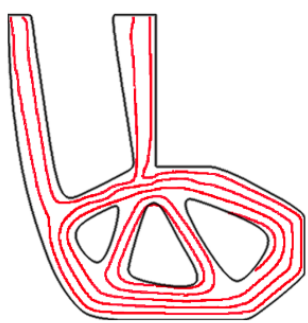

b

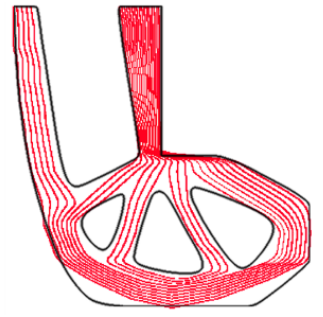

d

Figure 16. Printing paths of topology optimized L-shape part [36]: (a) applied point load and supports condition; (b) path generated by streamline method; (c) path generated by offset method; (d) path generated by EQS method.

The printing paths of topology optimized L-shape part by using streamline, offset and EQS methods are shown in Figure 16 (b), (c) and (d). As discussed above, the offset printing path can fill the whole 
structure, but contains interrupted paths and sharp corners. For the EQS printing path, there are lots of blank areas between the paths and the geometrical edges, and the paths are non-uniformly distributed which causes excessive material depositing in the high-density path areas. The streamline printing path has the problem of shape distortion which occurs mainly around holes and disrupts the continuity of stress transmission paths. The LPP printing path of topology optimized L-shape part is shown in Figure 17 (a). Evidently, the LPP path follows the vectors of tensile and compressive stresses and fills the whole part with variable spacing of the adjacent paths. As a continuous printing path, there is only one starting point and one finishing point as marked in the figure. The red paths inside the printing area have been connected by the blue one, which is same as the three-point bending bridge and suspension parts. As shown in Figure 17 (b), the printed L-shape part by using continuous carbon fiber reinforced polyamide filaments has the same geometry to the LPP path.

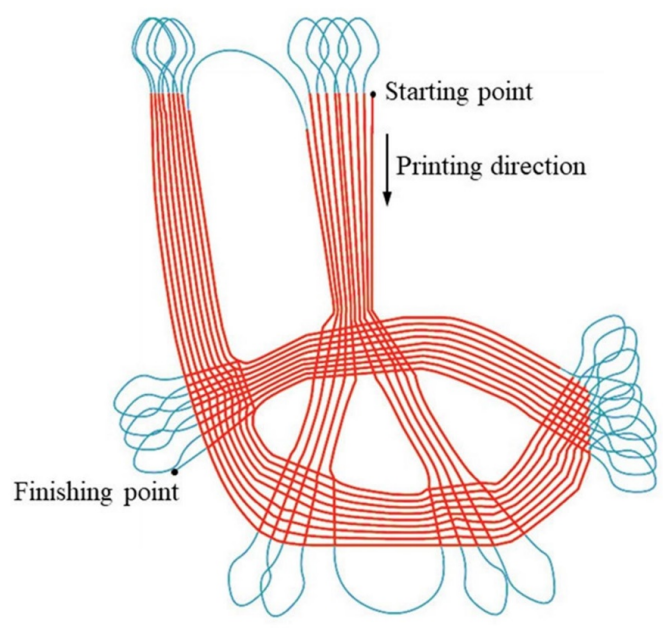

a

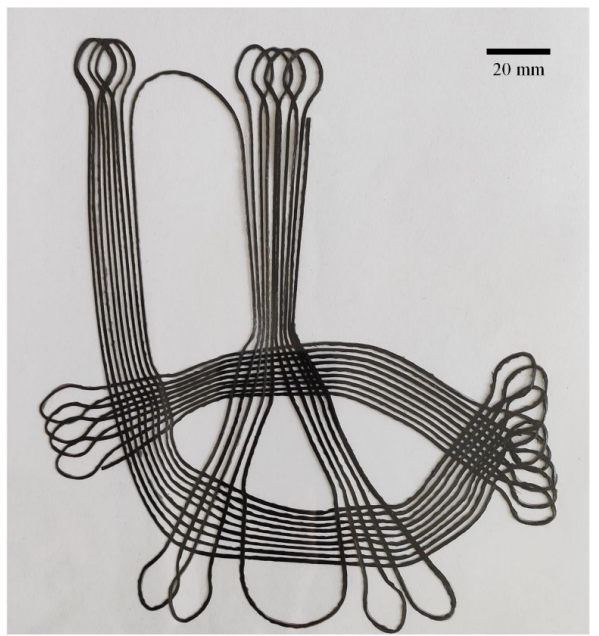

$\mathrm{b}$

Figure 17. (a) LPP generated printing paths of topology optimized L-shape part; (b) printed L-shape part with continuous carbon fiber reinforced polyamide filaments.

\section{Conclusion}

In this work, a load-dependent 3D printing path planning method of continuous fiber reinforced plastics has been proposed. By using the topological optimization methods, the load transmission paths are reordered and extracted. Then the developed Stress Vector Tracing algorithm has been used to generate the continuous load-dependent printing path of CFRPs from the extracted features, which contain geometry and stress vectors. Based on this method, the continuous fibers are printed along the load transmission path with high printing speed, variable spacing and low curvature turns. Several testing 
examples have been studied to verify the developed method. The result shows that the load-dependent 3D printing path planning method has the highest matching rate with the stress distribution. The state-of-theart path planning method for continuous fiber reinforced plastics have been compared with our method. No matter the offset or EQS methods show a defective geometry filling pattern and discontinuous printing path which cannot follow the load transmission. Lots of high curvature corners also prevent the availability of these paths. In future, the mechanical tests of the printed parts will be implemented to verify their mechanical properties.

\section{Acknowledgments}

The authors would like to thank the support of Alexander von Humboldt Foundation as well as to the donor, the German Federal Ministry for Education and Research.

\section{References}

[1] Wang X, Jiang M, Zhou Z, Gou J, Hui D. 3D printing of polymer matrix composites: A review and prospective. Composites Part B: Engineering. 2017;110:442-458.

[2] Brenken B, Barocio E, Favaloro A, Kunc V, Pipes RB. Fused filament fabrication of fiberreinforced polymers: A review. Additive Manufacturing. 2018;21:1-16.

[3] Agarwal K, Kuchipudi SK, Girard B, Houser M. Mechanical properties of fiber reinforced polymer composites: A comparative study of conventional and additive manufacturing methods. Journal of Composite Materials. 2018;52(23):3173-3181.

[4] Ning F, Cong W, Qiu J, Wei J, Wang S. Additive manufacturing of carbon fiber reinforced thermoplastic composites using fused deposition modeling. Composites Part B: Engineering. 2015;80:369-378.

[5] Baumann F, Scholz J, Fleischer J. Investigation of a new approach for additively manufactured continuous fiber-reinforced polymers. Procedia CIRP. 2017;66:323-328.

[6] Li N, Li Y, Liu S. Rapid prototyping of continuous carbon fiber reinforced polylactic acid composites by 3D printing. Journal of Materials Processing Technology. 2016;238:218-225.

[7] Li N, Link G, Jelonnek J. Rapid 3D microwave printing of continuous carbon fiber reinforced plastics. CIRP Annals. 2020. 
[8] Zhang H, Liu D, Huang T, Hu Q, Lammer H. Three-dimensional printing of continuous flax fiber-reinforced thermoplastic composites by five-axis machine. Materials. 2020;13(7):1-11.

[9] Tekinalp HL, Kunc V, Velez-Garcia GM, Duty CE, Love LJ, Naskar AK, et al. Highly oriented carbon fiber-polymer composites via additive manufacturing. Composites Science and Technology. 2014;105:144-150.

[10] Akhoundi B, Behravesh A. Effect of filling pattern on the tensile and flexural mechanical properties of FDM 3D printed products. Experimental Mechanics. 2019;59(6):883-897.

[11]Fidan I, Imeri A, Gupta A, Hasanov S, Nasirov A, Elliott A, et al. The trends and challenges of fiber reinforced additive manufacturing. The International Journal of Advanced Manufacturing Technology. 2019;102(5-8):1801-1818.

[12] Heidari-Rarani M, Rafiee-Afarani M, Zahedi A. Mechanical characterization of FDM 3D printing of continuous carbon fiber reinforced PLA composites. Composites Part B: Engineering. 2019;175:1-8.

[13] Dutra TA, Ferreira RTL, Resende HB, Guimarães A. Mechanical characterization and asymptotic homogenization of 3D-printed continuous carbon fiber-reinforced thermoplastic. Journal of the Brazilian Society of Mechanical Sciences and Engineering. 2019;41(3):1-15.

[14] Akhoundi B, Behravesh AH, Bagheri Saed A. An innovative design approach in threedimensional printing of continuous fiber-reinforced thermoplastic composites via fused deposition modeling process: In-melt simultaneous impregnation. Proceedings of the Institution of Mechanical Engineers, Part B: Journal of Engineering Manufacture. 2020;234(1-2):243-259.

[15] Mohammadizadeh M, Imeri A, Fidan I, Elkelany M. 3D printed fiber reinforced polymer composites-Structural analysis. Composites Part B: Engineering. 2019;175:1-6.

[16] Naranjo-Lozada J, Ahuett-Garza H, Orta-Castañón P, Verbeeten WM, Sáiz-González D. Tensile properties and failure behavior of chopped and continuous carbon fiber composites produced by additive manufacturing. Additive Manufacturing. 2019;26:227-241.

[17] Kousiatza C, Tzetzis D, Karalekas D. In-situ characterization of 3D printed continuous fiber reinforced composites: A methodological study using fiber Bragg grating sensors. Composites Science and Technology. 2019;174:134-141. 
[18] Ye W, Lin G, Wu W, Geng P, Hu X, Gao Z, et al. Separated 3D printing of continuous carbon fiber reinforced thermoplastic polyimide. Composites Part A: Applied Science and Manufacturing. 2019;121:457-464.

[19] Todoroki A, Oasada T, Mizutani Y, Suzuki Y, Ueda M, Matsuzaki R, et al. Tensile property evaluations of 3D printed continuous carbon fiber reinforced thermoplastic composites. Advanced Composite Materials. 2020;29(2):147-162.

[20]Ivey M, Melenka GW, Carey JP, Ayranci C. Characterizing short-fiber-reinforced composites produced using additive manufacturing. Advanced Manufacturing: Polymer \& Composites Science. 2017;3(3):81-91.

[21] Ming Y, Duan Y, Wang B, Xiao H, Zhang X. A novel route to fabricate high-performance 3D printed continuous fiber-reinforced thermosetting polymer composites. Materials. 2019;12(9):113.

[22] Liu T, Tian X, Zhang Y, Cao Y, Li D. High-pressure interfacial impregnation by micro-screw insitu extrusion for 3D printed continuous carbon fiber reinforced nylon composites. Composites Part A: Applied Science and Manufacturing. 2020;130:1-9.

[23] Quan C, Han B, Hou Z, Zhang Q, Tian X, Lu TJ. 3d printed continuous fiber reinforced composite auxetic honeycomb structures. Composites Part B: Engineering. 2020;187:1-12.

[24]Dickson AN, Barry JN, McDonnell KA, Dowling DP. Fabrication of continuous carbon, glass and Kevlar fibre reinforced polymer composites using additive manufacturing. Additive Manufacturing. 2017;16:146-152.

[25] Adumitroaie A, Antonov F, Khaziev A, Azarov A, Golubev M, Vasiliev VV. Novel Continuous Fiber Bi-Matrix Composite 3-D Printing Technology. Materials. 2019;12(18):1-10.

[26] De Backer W, Sinkez P, Chhabra I, Van Tooren MJ, Bergs A. In-Process Monitoring of Continuous Fiber Additive Manufacturing through Force/Torque Sensing on the Nozzle. AIAA Scitech 2020 Forum2020.

[27] Eichenhofer M, Wong JC, Ermanni P. Continuous lattice fabrication of ultra-lightweight composite structures. Additive Manufacturing. 2017;18:48-57.

[28] Liu S, Li Y, Li N. A novel free-hanging 3D printing method for continuous carbon fiber reinforced thermoplastic lattice truss core structures. Materials \& Design. 2018;137:235-244. 
[29] Shembekar AV, Yoon YJ, Kanyuck A, Gupta SK. Generating robot trajectories for conformal three-dimensional printing using nonplanar layers. Journal of Computing and Information Science in Engineering. 2019;19(3):1-13.

[30] Sugiyama K, Matsuzaki R, Malakhov AV, Polilov AN, Ueda M, Todoroki A, et al. 3D printing of optimized composites with variable fiber volume fraction and stiffness using continuous fiber. Composites Science and Technology. 2020;186. https://doi.org/10.1016/j.compscitech.2019.107905

[31] Li N, Link G, Wang T, Ramopoulos V, Neumaier D, Hofele J, et al. Path-designed 3D printing for topological optimized continuous carbon fibre reinforced composite structures. Composites Part B: Engineering. 2020;182. https://doi.org/10.1016/j.compositesb.2019.107612

[32] Fernandez-Vicente M, Calle W, Ferrandiz S, Conejero A. Effect of infill parameters on tensile mechanical behavior in desktop 3D printing. 3D printing and additive manufacturing. 2016;3(3):183-192.

[33] Sharma M, Rao IM, Bijwe J. Influence of fiber orientation on abrasive wear of unidirectionally reinforced carbon fiber-polyetherimide composites. Tribology International. 2010;43(5-6):959964.

[34] Bendsoe MP, Sigmund O. Topology optimization: theory, methods, and applications: Springer Science \& Business Media; 2013.

[35] Kenedi PP, Vignoli LL, Duarte BT, Matos FCdA, Dias HOT. Orthotropic Elastic Properties Assessment of Sandwich Laminates. Journal of Aerospace Technology and Management. 2017;9(3):389-396.

[36] Papapetrou VS, Patel C, Tamijani AY. Stiffness-based optimization framework for the topology and fiber paths of continuous fiber composites. Composites Part B: Engineering. 2020;183. https://doi.org/10.1016/j.compositesb.2019.107681

[37] Lee D-T. Medial axis transformation of a planar shape. IEEE Transactions on Pattern Analysis \& Machine Intelligence. 1982(4):363-369. 


\section{Figure and Table captions}

Figure 1. Different 3D printing methods of continuous fiber reinforced plastics: (a) out-of-nozzle impregnation; (b) in-nozzle impregnation and (c) semi-finished CFRP filament printing.

Figure 2. Schematic of the load-dependent path planning method.

Figure 3. Schematic of using Voronoi diagram to generate medial axis: (a) Voronoi diagram; (b) generated medial axis.

Figure 4. Geometrical parameters that are used in vector-trace algorithm.

Figure 5. Stresses analyzing of the bridge part: (a) mechanical analysis model; (b) stresses distribution after analyzing; (c) re-ordered stresses distribution inside the part.

Figure 6. Continuously connected printing path of the bridge part with variable printing speeds.

Figure 7. Processes of generating the continuous printing path of the scaled suspension part: (a) load and stresses distribution; (b) optimized stresses distribution and area; (c) main printing paths generated by the developed vector-trace algorithm; (d) generated continuous printing paths.

Figure 8. Curvature distribution of different printing paths of the bridge part: (a) to (d) curvature distribution of LPP, concentric, $45^{\circ}$ zigzag and $90^{\circ}$ zigzag printing paths.

Figure 9. Curvature distribution of different printing paths of the suspension part: (a) to (d) curvature distribution of the LPP, concentric, $45^{\circ}$ zigzag and $90^{\circ}$ zigzag printing paths.

Figure 10. Matching rate of different printing paths of the three-points-bending bridge part according to various angle difference of path and stress vectors.

Figure 11. Matching rate of different printing paths of the suspension part according to various angle difference of path and stress vectors.

Figure 12. Printing paths of topology optimized cantilever beam [36]: (a) load and supports condition; (b) path generated by streamline method; (c) path generated by offset method; (d) path generated by EQS method.

Figure 13. LPP generated printing paths of topology optimized cantilever beam: (a) main transfer directions of the stress distribution; (b) LPP generated printing path based on stress distribution. 
Figure 14. Printing paths of topology optimized M-shape part [36]: (a) applied point load and supports condition; (b) path generated by EQS method; (c) path generated by offset method; (d) path generated by streamline method.

Figure 15. LPP generated printing paths of topology optimized M-shape part: (a) main transfer directions of the stress distribution; (b) LPP generated printing path based on stresses distribution.

Figure 16. Printing paths of topology optimized L-shape part [36]: (a) applied point load and supports condition; (b) path generated by streamline method; (c) path generated by offset method; (d) path generated by EQS method.

Figure 17. (a) LPP generated printing paths of topology optimized L-shape part; (b) printed L-shape part with continuous carbon fiber reinforced polyamide filaments.

Table 1. Comparison of different printing path planning methods of CFRPs.

Table 2. Printing speeds according to the curvature, length of the curves and turning angle. 